LETTER TO THE EDITOR

\title{
Transmeatal microsurgery for intralabyrinthine and intrameatal schwannomas: literature review
}

\section{Approccio transmeatale microchirurgico nei neurinomi intralabirintici e intrameatali: revisione della letteratura}

\author{
Daniele Marchioni ${ }^{1}$, Flavia Di Maro', Livio Presutti² \\ ${ }^{1}$ Otolaryngology-Head and Neck Surgery Department, University Hospital of Verona, Verona, Italy; ${ }^{2}$ Otolaryngology-Head and Neck \\ Surgery Department, Alma Mater Studiorum - University of Bologna, Bologna, Italy
}

KEY WORDS: intracanalicular acoustic neuroma, transcanal approaches, internal auditory canal, transpromontorial corridor

PAROLE CHIAVE: neurinoma dell'acustico intracanalare, approcci transcanalari, condotto uditivo interno, via transpromontoriale

\section{Dear Editor,}

We have carefully read a recent issue published by Acta Otorhinolaryngologica Italica written by Mazzoni et al. ${ }^{1}$ who intends to reconsider the transcanal transvestibular approach to the labyrinth and Internal Auditory Canal (IAC) for the treatment of acoustic neuroma. We have some considerations to make in this regard.

In 1970, Alvarez de Cozar et al. described a transcanal transvestibular approach for the management of disabling unilateral Ménière's disease. As stated later by the authors, the aims of this approach were: to perform a labyrinthectomy exploring both the Internal Auditory Canal (IAC) and the cochlea in order to exclude the presence of an undiagnosed intralabyrinthine neuroma, to provide a diagnostic tool for neuromas requiring labyrinthectomy for removal and for treatment of small $<3 \mathrm{~mm}$, mobile and unexpected intracanalicular neuromas ${ }^{2}$. In 1975, the same authors reported their experience with this approach on 178 patients with intractable vertigo; in three of them an intracanalicular neuroma was found. In two of the patients the tumour was removed with a transvestibular approach, and in one case it was necessary to perform a combined transvestibular and translabyrinthine approach ${ }^{2}$. In the conclusions, the authors specified that "In the case of a small $<3 \mathrm{~mm}$, unexpectedly found, acoustic neurinoma which is freely mobile within the canal, removal can be attempted through this approach. We consider removal of larger tumours by this approach hazardous for the preservation of the facial nerve function and do not recommend it under these circumstances" ${ }^{2}$.

In 2015, we presented the Endoscopic Transcanal Transpromontorial approach ${ }^{3}$, the first transcanal transpromontorial endoscopic approach to the IAC. With this approach it is possible to dominate the IAC through the promontory, preserving the medial wall of the vestibule. Actually, in the surgical approach, after transcanal exposure of the vestibule, spherical recess and basal, middle and apical turn of the cochlea, drilling is performed around the inferior aspect of the vestibule until the fundus of the IAC is opened, exposing the most lateral portion of the pathology. The IAC is increasingly exposed by further drilling around the inferior aspect of the vestibule. The limits of the dissection are the tympanic segment of the facial nerve superiorly, the internal carotid artery anteriorly, the jugular bulb inferiorly and the mastoid portion of the facial nerve posteriorly. The anterior, inferior and posterior borders of
Received: February 1, 2021

Accepted: February 3, 2021

\author{
Correspondence \\ Flavia Di Maro \\ Otolaryngology-Head and Neck Surgery De- \\ partment, University Hospital of Verona \\ piazzale Aristide Stefani 1, 37126 Verona, Italy \\ Tel. +39045 8122330. Fax +390458122313 \\ E-mail: flaviadimaro@yahoo.com
}

Funding

None.

Conflict of interest

The Authors declare no conflict of interest.

How to cite this article: Marchioni D, Di Maro F, Presutti L. Transmeatal microsurgery for intralabyrinthine and intrameatal schwannomas: literature review. Acta Otorhinolaryngol Ital 2021;41:282-284. https://doi. org/10.14639/0392-100X-N1443

(c) Società Italiana di Otorinolaringoiatria e Chirurgia Cervico-Facciale

\section{(c) (1) $(2)$}

This is an open access article distributed in accordance with the CC-BY-NC-ND (Creative Commons Attribution-NonCommercial-NoDerivatives 4.0 International) license. The article can be used by giving appropriate credit and mentioning the license, but only for non-commercial purposes and only in the original version. For further information: https:// creativecommons.org/licenses/by-nc-nd/4.0/deed.en 
the IAC are exposed by drilling in a circumferential manner under the vestibule and around the IAC in a horseshoe shape, deepening from lateral to medial until reaching the reflection of the dura from the IAC meatus to the medial surface of the petrous bone. Subsequently, the IAC dura is opened to expose the tumour. The acoustic neuroma is then removed from the meatus to the fundus of the IAC, preserving the facial nerve. When extension to the cerebellopontine angle (CPA) is found, additional bone is drilled to enlarge the opening of the IAC meatus to allow management of the residual tumour lying in the CPA. This drilling is performed by following the acoustic-facial bundle toward the entry zone ${ }^{4}$.

In our experience, the indications of this approach consist of disease involving the cochlea, vestibule and the IAC including minimal extension in the CPA (acoustic neuroma Koos I-II). At the end of surgery, depending on the extent of the disease and the extent of tissue extirpation, either repositioning of the tympanomeatal flap or a cul-de-sac closure of the skin of the ear canal can be performed ${ }^{3}$. In the last few years this approach has been used in other departments worldwide ${ }^{5}$.

Later ${ }^{6}$ we developed a microscopic approach through the same route, the Expanded Transcanal Transpromontorial approach. It enlarges the surgical window to the CPA allowing resection of acoustic neuroma previously resectable only through more invasive approaches. The Expanded Transcanal Transpromontorial approach allows complete domination of the IAC and the corresponding portion of CPA and is specifically indicated for the management of acoustic neuroma up to selected Koos III ${ }^{7}$. As for the endoscopic approach, in this microscopic approach access to the IAC occurs through a transpromontorial route, preserving the medial wall of the vestibule. Both techniques therefore represent an absolute innovation in the panorama of approaches to the IAC and currently represent acknowledged options in the surgical treatment of neuromas previously removable only through retrosigmoid, middle cranial fossa or translabyrinthine approaches. The choice between these two transcanal approaches is made based on tumour extent: if the tumour is completely located in the IAC with or without minimal CPA involvement (Koos stages I-II), a totally transcanal endoscopic exclusive approach can be performed; if the tumour occupies the IAC involving the CPA in a straight line as far as the entry zone to the brain stem (Koos stages II-III), an Expanded Transcanal Transpromontorial approach is preferred.

Mazzoni et al. ${ }^{1}$ presented a case series of eight patients diagnosed with intralabyrinthine schwannoma and/or intrameatal vestibular schwannoma operated through a transmeatal microsurgical approach. The authors stated that the technique was described 50 years ago, apparently ignoring the aim and possible applications of the approach described by Alvarez de Cozar et al. ${ }^{2}$.

In the case series, most of the articles describe transcanal approaches for neuromas limited to the vestibule or with minimal extension to the cochlea, without access the IAC. DeLozier ${ }^{8}$ in a case of intractable vertigo, accesses the IAC for exploration purposes, finding no pathology; Jiang et al. ${ }^{9}$ described 2 cases of intracochlear neuroma without extension to the vestibule or IAC treated with a retroauricolar transotic transcanal approach, recommending the traditional transmastoid transotic approach for tumours with extension to the IAC; finally, Zhu et al. ${ }^{10}$ presented a case report of an intracochlear neuroma with extension to the modiolus.

None of the articles in the case series describe an approach allowing removal of tumours occupying the entire IAC (as opposed to the case reported in the main paper) with or without extension into the CPA.

Furthermore, it is surprising how the author does not consider in the literature review our large series of 49 patients who underwent Endoscopic Transcanal Transpromontorial approach (21 cases) or Expanded Transcanal Transpromontorial approach (28 cases) for acoustic neuroma Koos I-III. To the best of our knowledge, this is the largest case series of transcanal transpromontorial approach for intrameatal acoustic neuroma and in clinical practice there are today more than 100 procedures. We consider a serious shortcoming that an Italian team does not consider in its review an approach developed by another Italian team.

In conclusion, the Expanded Transcanal Transpromontorial approach is an innovative approach for the management of intracanalicular acoustic neuroma up to some Koos III tumour, first described in $2017^{6}$. Although indications are limited to growing tumours with impaired hearing, surgical experience in these minimally invasive approaches is increasing, allowing the removal of acoustic neuromas previously approachable only through more invasive techniques.

\section{References}

1 Mazzoni A, Zanoletti E, Cazzador D, et al. Transmeatal microsurgery for intralabyrinthine and intrameatal schwannomas: a reappraisal. Acta Otorhinolaryngol Ital 2020;40:390-395. https://doi. org/10.14639/0392-100X-N0779

2 Alvarez de Cozar F, Antoli-Candela F. Transvestibular approach to the internal auditory canal. Ann Otol Rhinol Laryngol 1975;84:145-51. https://doi.org/10.1177/000348947508400201

3 Marchioni D, Alicandri-Ciufelli M, Rubini A, et al. Endoscopic transcanal corridors to the lateral skull base: initial experiences. Laryngoscope 2015;125(Suppl 5):S1-13. https://doi.org/10.1002/lary.25203

4 Marchioni D, Alicandri-Ciufelli M, Rubini A, et al. Exclusive endoscopic transcanal transpromontorial approach: a new perspective for 
internal auditory canal vestibular schwannoma treatment. J Neurosurg 2017;126:98-105. https://doi.org/10.3171/2015.11.JNS15952

5 Wick CC, Arnaoutakis D, Barnett SL, et al. Endoscopic transcanal transpromontorial approach for vestibular schwannoma resection: a case series. Otol Neurotol 2017;38:e490-e494. https://doi. org/10.1097/MAO.0000000000001588

6 Presutti L, Alicandri-Ciufelli M, Bonali M, et al. Expanded transcanal transpromontorial approach to the internal auditory canal: pilot clinical experience. Laryngoscope 2017;127:2608-2614. https://doi. org/10.1002/lary.26559

7 Marchioni D, Soloperto D, Masotto B, et al. Transcanal transpromontorial acoustic neuroma surgery: results and facial nerve out- comes. Otol Neurotol 2018;39:242-249. https://doi.org/10.1097/ MAO.0000000000001658

8 DeLozier HL, Gacek RR, Dana ST. Intralabyrinthine schwannoma. Ann Otol Rhinol Laryngol 1979;88:187-191. https://doi. org/10.1177/000348947908800207

9 Jiang ZY, Kutz JW Jr, Roland PS, et al. Intracochlear schwannoma confined to the otic capsule. Otol Neurotol 2011;32:117-119. https:// doi.org/10.1097/MAO.0b013e31822a20ea

10 Zhu AF, McKinnon BJ. Transcanal surgical excision of an intracochlear schwannoma. Am J Otolaryngol 2012;33:779-781. https://doi. org/10.1016/j.amjoto.2012.07.007 